POSTER 13-23

\section{Red Cabin Lights Impair Flight Crew Performance of Color-Dependent Tasks}

*David Hightower, MD, Stephen H. Thomas, MD, C. Keith Stone, MD, Susan Brinkley, RN

Division of Air Medical Services, Department of Emergency Medicine, East Carolina University School of Medicine, Greenville, North Carolina

Purpose: Prehospital transport commonly is performed in helicopters lacking partition between the pilot and medical crew cabin spaces. For safety reasons, nighttime cabin lighting in these aircraft must be red. This study was conducted to determine if red lighting impairs the ability of the air medical crew to perform the following tasks: 1) reading red-colored medication labels; 2) interpreting colorimetric; 3) end-tidal $\mathrm{CO}_{2}$ detectors; and 4) identifying cyanosis.

Methods: In dark conditions, an EMS instructor pilot adjusted red lighting in a B-105 helicopter to maximum acceptable intensity. $A$ total of 13 emergency medicine residents were shown positive and negative $\mathrm{CO}_{2}$ detectors, and skin-colored and cyan-tinted rubber masks. The percentages of correctly identified masks and detectors were noted. Subjects also read the drug name and quantity from 12 medication labels. Wilcoxon analysis compared label-reading accuracy between two groups: control (black and white lettering or background) and red (red lettering or background).

Results: The accuracy of mask and $\mathrm{CO}_{2}$ detector identification in a normal-lighted setting was $100 \%$ : the percentages of accurately identified masks and detectors in the red-light setting are listed in the table below.

$\begin{array}{ccccc} & \begin{array}{c}\text { normal } \\ \text { mask }\end{array} & \begin{array}{c}\text { cyanotic } \\ \text { mask }\end{array} & \begin{array}{c}\text { negative } \\ \mathbf{C O}_{2}\end{array} & \begin{array}{c}\text { positive } \\ \mathbf{C O}_{2}\end{array} \\ \% \text { Identified } & 61.5 \% & 30.8 \% & 46.2 \% & 46.2 \%\end{array}$

Ability to correctly read medication labeling was significantly $(p=0.003)$ greater in control $(76.9 \pm 10.9 \%)$ as compared to red groups $(16.3 \pm 13.4 \%)$.

Conclusion: Red cabin lighting appears to impair air medical crew performance of certain potentially critical tasks requiring color discernment.
POSTER 14-29

\section{Standing Orders Versus Call-in Orders: Effect on Prehospital Times}

\author{
*Stephen C. Ausband, BS, MS-III, Lawrence \\ Brown, EMT-P, N. Heramba Prasad, MD, \\ Barbara McMahon, RN, EMT-P \\ Division of EMS, Department of Emergency Medicine, \\ East Carolina University School of Medicine, Greenville, \\ North Carolina
}

Hypothesis: Calls utilizing standing orders have shorter total prehospital times than do those using call-in orders.

Methods: One-hundred-thirty-three ambulance call reports were reviewed over a two-month period for patients with chest pain (CP) and shortness of breath (SOB). During the first month, both types of calls required call-in orders. During the second month, the $\mathrm{CP}$ protocols allowed the use of standing orders for the administration of ALS skills, while SOB calls still required a call-in order. Results were compared for total prehospital and on-scene times using Student's $t$-test.

Results: A total of 133 reports yielded 38 standing orders and 95 call-in orders. The difference in scene time between when standing orders and call-in orders were used was significant ( $14.2 \mathrm{~min}$ vs. $18.0 \mathrm{~min}, p<.005$ ). The total prehospital time also was significantly shorter for patients treated using standing orders versus call-in orders (20.2 $\mathrm{min}$ vs. $25.7 \mathrm{~min}, p<.005$ ). The differences in time for calls requiring ALS interventions on which medicines and IVs were started showed no significant difference in scene or total prehospital times when comparing standing orders versus call-in orders. There was no significant difference in times for calls requiring only that medicines be given or IVs started. There was no difference between times for $\mathrm{CP}$ or SOB calls in either month.

Conclusion: The lack of difference in time between callin orders and standing orders during ALS calls suggests that the majority of prehospital time is spent performing ALS skills and not calling for orders. These data suggest that standing orders do not save time. 